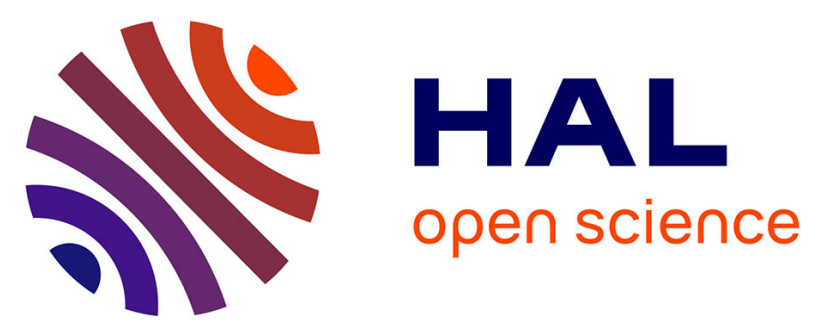

\title{
High energy PIXE: A tool to characterize multi-layer thick samples
}

\author{
A. Subercaze, Charbel Koumeir, Vincent Métivier, Noël Servagent, Arnaud \\ Guertin, Ferid Haddad
}

\section{- To cite this version:}

A. Subercaze, Charbel Koumeir, Vincent Métivier, Noël Servagent, Arnaud Guertin, et al.. High energy PIXE: A tool to characterize multi-layer thick samples. Nuclear Instruments and Methods in Physics Research Section B: Beam Interactions with Materials and Atoms, 2018, 417, pp.41-45. 10.1016/j.nimb.2017.09.009 . hal-02912545

\section{HAL Id: hal-02912545 \\ https://hal.science/hal-02912545}

Submitted on 29 Jan 2021

HAL is a multi-disciplinary open access archive for the deposit and dissemination of scientific research documents, whether they are published or not. The documents may come from teaching and research institutions in France or abroad, or from public or private research centers.
L'archive ouverte pluridisciplinaire HAL, est destinée au dépôt et à la diffusion de documents scientifiques de niveau recherche, publiés ou non, émanant des établissements d'enseignement et de recherche français ou étrangers, des laboratoires publics ou privés. 


\title{
High energy PIXE : a tool to characterize multi-layer thick samples
}

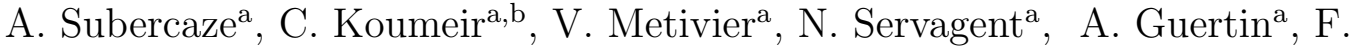 \\ Haddad $^{a, b}$ \\ ${ }^{a}$ Laboratoire SUBATECH, Institut Mines Télécom Atlantique, Université de Nantes, \\ CNRS/IN2P3, 4 Rue Alfred Kastler, 44307 Nantes cedex 3 - FRANCE \\ ${ }^{b}$ GIP ARRONAX, 1 rue ARONAX, 44817 Saint-Herblain cedex - FRANCE
}

\begin{abstract}
High energy PIXE is a useful and non-detructive tool to characterize multilayer thick samples such as cultural heritage objects. In a previous work, we demonstrated the possibility to perform quantitative analysis of simple multi-layer samples using high energy PIXE, without any assumption on their composition. In this work an in-depth study of the parameters involved in the method previously published is proposed. Its extension to more complex samples with a repeated layer is also presented. Experiments have been performed at the ARRONAX cyclotron using $68 \mathrm{MeV}$ protons. The thicknesses and sequences of a multi-layer sample including two different layers of the same element have been determined. Performances and limits of this method are presented and discussed.
\end{abstract}

Keywords: High energy PIXE; Ion beam analysis; Multi-layers; $\frac{K_{\alpha}}{K_{\beta}}$ ratio.

\section{Introduction}

Analysis of cultural heritage objects is a very active application field for the PIXE method [1-3], especially thanks to its ability to determine their elemental composition in a non-destructive way. In many cases, those objects consist of a stack of many layers (paintings, coins...) with sometimes several layers made of the same element. Differential PIXE [4], using low ernergy beam, was developped in order to provide depth concentration profile, composition and ordering of the layers. The principle of this technique relies on the comparison between PIXE spectra recorded at different beam energies 
[5] (e.g from 1 to $4 \mathrm{MeV}$ ) or at different incident angles [6]. Complementary information about the target is obtained for each measurement due to the strong variation of the ionization cross sections, at low energy, within the target. It is, therefore, possible to use a de-convolution algorithm to extract the contribution of each layer. Differential PIXE has been used to study multi-layer samples [7] with a thickness up to tens of microns [8].

High-energy PIXE (HEPIXE) is suitable to perform non destructive analysis of thick samples, in normal air, due to the high penetration range of energetic light ions in matter and their ability to excite the energetic $\mathrm{K} \mathrm{X}$ rays. HEPIXE has already been used in the field of cultural heritage [9-11] and especially for the characterization of ancient paintings with several superposed layers [12]. Metallic archeological objects can be also investigated without removing the patina layer present on their surface. The investigated thicknesses in those works were between few dozens and few hundred of micrometers. But in this case, contrary to the usual differential PIXE method, ionization cross sections change smoothly with the energy and incident angle variation. However, HEPIXE analysis using $\frac{K_{\alpha}}{K_{\beta}}$ (or $\frac{K_{\alpha}}{L_{\alpha}}, \frac{L_{\alpha}}{L_{\beta}}$ ) ratio provides qualitative information about the thicknesses and the sequences of several layers but assumptions on the sample composition is required [12]. In a recent work [13], we demonstrate the possibility to perform quantitative analysis of simple multi-layer samples without any assumption on their composition. This method, based on the relative variation of $\frac{K_{\alpha}}{K_{\beta}}\left(\right.$ or $\frac{K_{\alpha}}{L_{\alpha}}, \frac{L_{\beta}}{K_{\alpha}}$ ) when the sample is rotated, had given good results for patterns of different pure material foils. But in more realistic applications, there might be some repetition of a layer inside the samples. The present work aims at studying samples with repeteated layers based also on the relative variation of $\frac{K_{\alpha}}{K_{\beta}}$ (or $\left.\frac{K_{\alpha}}{L_{\alpha}}, \frac{L_{\beta}}{K_{\alpha}}\right)$ ratio. This study is an extention of the previous method [13] and it is a step further to develop an acurate quantitative method in order to analyze cultural heritage samples later on. Firstly the experimental setup is described. Secondly, the key parameters of the method such as the self attenuation and the impact of the layer placed in front of the emitting one are described. Finally, the extension of the previous method [13] to the analysis of a multi-layer sample with a repeated layer is presented. 


\section{Experimental procedure}

\subsection{Experimental set-up}

High energy experiments were carried out at ARRONAX cyclotron [14] using $68 \mathrm{MeV}$ protons. The beam diameter on the sample was in the range of $1 \mathrm{~cm}$. The X-rays are detected by a High Purity Germanium (HPGe) detector. The distance between the sample and the detector is $25 \mathrm{~cm}$. The sample holder can be rotated with respect to the incident beam in order to change the angle between the sample and the detector, with a precision of $0.01^{\circ}$. An ionization chamber is used in order to monitor the number of particles penetrating the samples. The ionization chamber current is measured by a high precision commercial electrometer (MULTIDOS-PTW). Prior to the samples irradiations, the ionization chamber is calibrated using a faraday cup. During the experiments, the beam intensity is kept around $100 \mathrm{pA}$ and the irradiations last about 15 minutes. The high energy PIXE platform is described in more details in [15].

\subsection{Studied samples}

The composition and the thickness of the samples prepared for this work are listed in table 1. The samples are composed by a stack of several pure material foils. Those foils are provided by GoodFellow and their dimensions are $25 \times 25 \mathrm{~mm}^{2}$. The Al-Ti sample is used to study the linearity of $\Delta \mu \cdot d$ (see section 3.2) involved in the determination of the layers thicknesses. The $\mathrm{Ti}-\mathrm{Ag}_{1}-\mathrm{Au}_{\mathrm{Ag}} \mathrm{Ag}_{2}$ is prepared as a multi-layer sample with a repeated layer in order to perform the associated analysis. References samples for each elements are also irradiated using the same experimental configuration as the $\mathrm{Ti}-\mathrm{Ag}_{1}-\mathrm{Au}-\mathrm{Ag}_{2}$ irradiation. Those reference samples are used to reduce the experimental errors (such as detection efficiency) on the quantification of the layers thicknesses.

Table 1: Composition of the irradiated samples for this study. The value in the parenthesis is the thickness of the given pure foil.

\begin{tabular}{|c|c|}
\hline Sample name & Composition \\
\hline $\mathrm{Al}-\mathrm{Ti}$ & $\mathrm{Al}(20 \mu \mathrm{m}) / \mathrm{Ti}(10 \mu \mathrm{m})$ \\
$\mathrm{Ti}-\mathrm{Ag}_{1}-\mathrm{Au}-\mathrm{Ag}_{2}$ & $\mathrm{Ti}(10 \mu \mathrm{m}) / \mathrm{Ag}(10 \mu \mathrm{m}) / \mathrm{Au}(10 \mu \mathrm{m}) / \mathrm{Ag}(25 \mu \mathrm{m})$ \\
\hline \hline $\mathrm{Ti}_{\text {ref }}$ & $\operatorname{Ti}(10 \mu \mathrm{m})$ \\
$\mathrm{Ag}_{r e f}$ & $\operatorname{Ag}(10 \mu \mathrm{m})$ \\
$\mathrm{Au}_{r e f}$ & $\mathrm{Au}(10 \mu \mathrm{m})$ \\
\hline
\end{tabular}


The thicknesses of the foils presented in table 1 are given by GoodFellow. Acurate values obtained using a scanned and a high precision scale for the $\mathrm{Ti}$ of $\mathrm{Ti}-\mathrm{Ag}_{1}-\mathrm{Au}-\mathrm{Ag}_{2}$ are presented in table 4.

\subsection{Multi-layer analysis method}

The analysis method [13] is based on the relative variation of $\frac{K_{\alpha}}{K_{\beta}}$ (or $\frac{K_{\alpha}}{L_{\alpha}}$, $\left.\frac{L_{\beta}}{K_{\alpha}}\right)$ ratio $\left(K_{\alpha}\right.$ is the number of detected $K_{\alpha}$ X-rays), named $\frac{\Delta R}{R}$, as a function of the angle between the target and the detector, $\theta=0$ when the detector axis is normal to the sample, i.e their surfaces are parallel. This relative variation is given by :

$$
\frac{\Delta R}{R}=\frac{R\left(\theta_{1}\right)-R\left(\theta_{2}\right)}{R\left(\theta_{1}\right)}=1-f_{\text {self }} \cdot \frac{e^{\left(\frac{\Delta \mu \cdot d}{\cos \left(\theta_{2}\right)}\right)}}{e^{\left(\frac{\Delta \mu \cdot d}{\cos \left(\theta_{1}\right)}\right)}},
$$

where $\Delta \mu=\mu_{K_{\beta}}-\mu_{K_{\alpha}}$ with $\mu$ the attenuation coefficient [16] of the layers placed in front of the emitting one, $\mathrm{d}$ is X-rays path in this layer and $f_{\text {self }}$ is given by the equation :

$$
f_{\text {Self }}=\frac{\left(\frac{1-e^{\left(\frac{-\mu_{\alpha}^{\prime} \cdot d^{\prime}}{\cos \left(\theta_{2}\right)}\right)}}{1-e^{\left(\frac{-\mu_{\beta}^{\prime} \cdot d^{\prime}}{\cos \left(\theta_{2}\right)}\right)}}\right)}{\left(\frac{1-e^{\left(\frac{-\mu_{\alpha}^{\prime} \cdot d^{\prime}}{\cos \left(\theta_{1}\right)}\right)}}{1-e^{\left(\frac{-\mu_{\beta}^{\prime} \cdot d^{\prime}}{\cos \left(\theta_{1}\right)}\right)}}\right)},
$$

where $\mu^{\prime}$ is the attenuation coefficient of the emitting layer and $d^{\prime}$ the X-rays path inside it. The function $f_{\text {self }}$ represents the ratio of the $\frac{K_{\alpha}}{K_{\beta}}$ attenuation in the emitter layer itself, in other words the self attenuation. The impact of this factor is presented in the next section.

When $f_{\text {self }}$ is negligible, we can extract from equation 1 :

$$
\Delta \mu \cdot d=\frac{1}{\frac{1}{\cos \left(\theta_{2}\right)}-\frac{1}{\cos \left(\theta_{1}\right)}} \cdot \ln \left(1-\frac{\Delta R}{R}\right) .
$$

$\Delta \mu \cdot d$ contains the attenuation of the effective layer located between the emitting one and the detector (the effective layer has the same effect on the $\mathrm{X}$-ray attenuation than real layers). Using the photoelectric mass attenuation 
coefficient (far from the shell edge), $\frac{\mu}{\rho}$ given in [17] and equation 3 we can calculate the attenuation of $K_{\alpha}$ lines (or $\left.K_{\beta}, L_{\alpha}, L_{\beta}\right) M_{K_{\alpha}}$

$$
M_{K_{\alpha}}=\sum_{i=0}^{n} e^{\left(-\mu_{\alpha}^{i} \cdot d^{i}\right)}=e^{-\Delta \mu \cdot d \cdot\left(\frac{\frac{1}{\left(E_{K_{\alpha}}\right)^{7 / 2}}}{{\left(E_{K_{\beta}}\right)^{7 / 2}-\frac{1}{\left(E_{K_{\alpha}}\right)^{7 / 2}}}^{-}}\right)},
$$

where $\mathrm{n}$ is the number of layers placed in front of the emitting one and $\mathrm{E}$ the energy of the considered X-ray. The detected number of $K_{\alpha}$ X-rays (or $K_{\beta}$, $\left.L_{\alpha}, L_{\beta}\right), N_{K \alpha}^{\text {layer }}$, can then be corrected from this attenuation . Therefore we can determine the thickness of a layer, $e_{\text {layer }}$

$$
e_{\text {layer }}=-\frac{1}{\mu_{\alpha}^{\prime}} \cdot \ln \left(1-\frac{N_{K_{\alpha}}^{\text {layer }}}{N_{K_{\alpha}}^{\text {Standard }} \cdot M_{K_{\alpha}}}\right),
$$

where $N_{K_{\alpha}}^{\text {Standard }}$ is the X-ray intensity of a standard sample irradiated in the same condition.

To determine the sequence of a multi-layer sample, $\Delta \mu \cdot d$ can't be used because of its dependency on the energy of the X-rays. Using the definition of $\frac{\mu}{\rho}$ given in [17], we can calculate a new term $(\Delta \mu \cdot d)^{\prime}$ defined by equation 6.

$$
(\Delta \mu \cdot d)^{\prime}=\frac{1}{\frac{1}{E_{K_{\beta}}^{7 / 2}}-\frac{1}{E_{K_{\alpha}}^{7 / 2}}} \cdot \frac{1}{\frac{1}{\cos \left(\theta_{2}\right)}-\frac{1}{\cos \left(\theta_{1}\right)}} \cdot \ln \left(1-\frac{\Delta R}{R}\right)
$$

$(\Delta \mu \cdot d)^{\prime}$ is also equal to $: \rho \cdot d \cdot Z^{5} \cdot \frac{N_{a}}{A}$, where $E$ is the energy of the considered X-ray, $N_{A}$ is the Avogadro constant and $Z, A, \rho, \mathrm{d}$ are the effective atomic number, mass number, density and thickness of the layers placed in front of the emitting one. The comparison between $(\Delta \mu \cdot d)^{\prime}$ values for each detected elements allows to determine the sequences of the layers (demonstrated in $[13])$.

\section{Robustness of the method}

In this section, the main parameters (described in the previous section) of the analysis method are studied. 


\subsection{Self attenuation}

The relative variation of $\mathrm{R}, \frac{\Delta R}{R}$, is function of the self attenuation and of the attenuation inside the layers placed above the emitting one. In order to use $\frac{\Delta R}{R}$ to determine the thickness and the position of the emitting layer, the self attenuation factor has to be small compared to the attenuation inside the layers. The variation of the self attenuation factor, $f_{\text {self }}$, as a function of the emitting layer thickness (g.cm ${ }^{-2}$ ) and for $\theta_{1}=45^{\circ}$ and $\theta_{2}=0^{\circ}$ is shown in figure 1. These angles have been choosen because they lead to the maximum variation of the self attenuation factor (i.e the worst case).

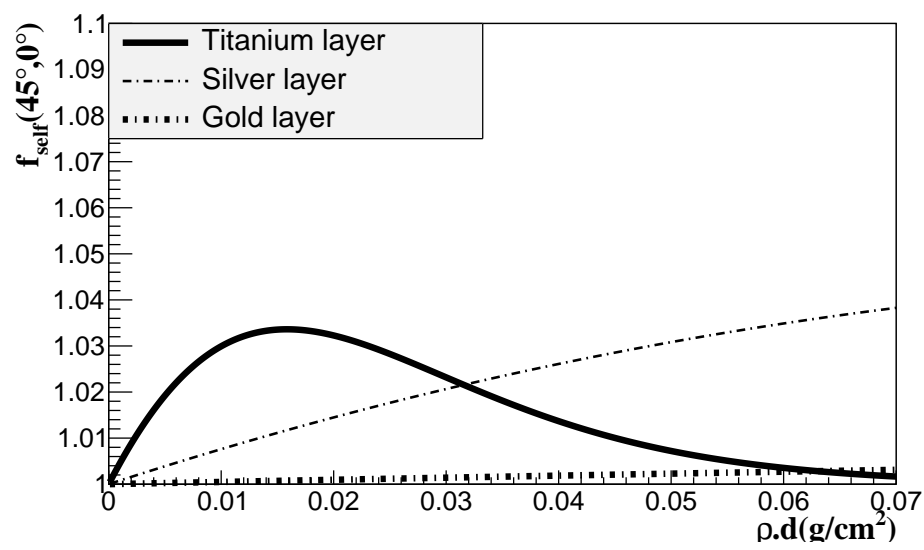

Figure 1: $f_{\text {self }}$ as a function of $\rho \cdot d$ calculated using equation 2 for titanium, silver and gold and for $\theta_{1}=45^{\circ}$ and $\theta_{2}=0^{\circ}$. The self attenuation factor is calculated for the $\frac{K_{\alpha}}{K_{\beta}}$ ratio.

For these three elements ( $\mathrm{Ti}, \mathrm{Ag}$ and $\mathrm{Au}$ representing a wide range of $\mathrm{Z}$ ), the self attenuation factor is less than 1.04.

For the titanium, due to its low energy X-ray, even a low amount of light element in front of it will induce a value of $\frac{\Delta R}{R}$ far larger than 1.04. For example, $20 \mu \mathrm{m}$ of aluminium gives $\frac{\Delta R}{R}=1.15$ for the titanium. So the self attenuation is negligeable and is not an issue for light element.

In the silver case, a low value of $\frac{\Delta R}{R}(1-3 \%)$ can be due to the self attenuation of a thick $\mathrm{Ag}$ layer or to the attenuation by the layers above in case of a thin Ag layer. We can solve this issue using the value of the $K_{\alpha}$ intensity. In fact a thick layer of $\mathrm{Ag}$ will induce a higher $K_{\alpha}$ intensity compared to a thin one. 
In the gold case, the self attenuation factor is lower than 1.01, but in the majority of the cases the $\frac{K_{\alpha}}{K_{\beta}}$ ratio will not vary so much due to the high energy K X-rays. Therefore this ratio cannot be used (or in very specific cases like very thick samples). $\frac{L_{\alpha}}{K_{\alpha}}$ or $\frac{L_{\beta}}{K_{\alpha}}$ ratios can be used instead. The variation of $f_{\text {self }}$ in this case is represented in figure 2. For the couple of angles $\left(45^{\circ}\right.$, $0^{\circ}$ ), the self attenuation factor is too high (up to 1.35) but by choosing a smaller angle variation $\left(25^{\circ}\right)$ we can reduce $f_{\text {self }}$. Due to the low energy of the L X-rays, even for a narrowed angular range, the angular variations of those ratio are sensitive to thin layer (few dozens of $\mu \mathrm{m}$ ) contrary to the $\frac{K_{\alpha}}{K_{\beta}}$ ratio. Therefore for heavy elements we can use the $\frac{L_{\alpha}}{K_{\alpha}}$ (or $\frac{L_{\beta}}{K_{\alpha}}$ ) ratio and a narrowed angular range to determine the sequences and the thicknesses with a negligible influence of the self attenuation.

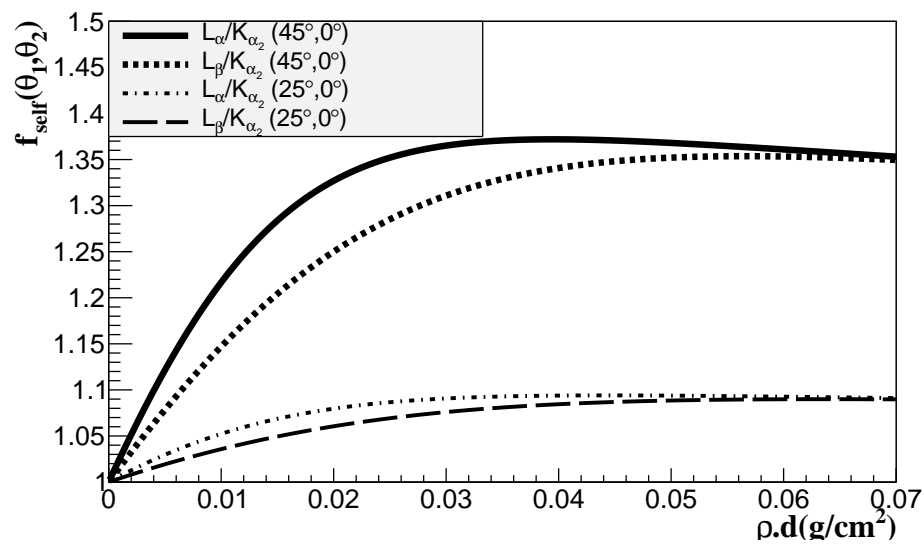

Figure 2: Self attenuation factor as a function $\rho \cdot d$ calculated using equation 2 for the gold and for $\theta_{1}=45$ or $25^{\circ}$ and $\theta_{2}=0^{\circ}$. The self attenuation factor is calculated for the $\frac{L_{\alpha}}{K_{\alpha}}$ and $\frac{L_{\beta}}{K_{\alpha}}$ ratio.

Looking at the self attenuation factor for different elements, we have defined a framework for the method. Analysis of light elements is not affected by the self attenuation. For mid-Z elements and low value of $\frac{\Delta R}{R}$, the value of the $K_{\alpha}$ intensity has to be considered. For heavy elements, the ratio $\frac{L_{\alpha, \beta}}{K_{\alpha}}$ has to be used instead of the $\frac{K_{\alpha}}{K_{\beta}}$ with a lower angles $\left(\theta_{2^{-}} \theta_{1}\right)$.

\subsection{Attenuation in layers placed in front of the emetting layer $: \Delta \mu \cdot d$}

The normalization of $\Delta \mu \cdot d$ by $\frac{1}{\cos \left(\theta_{2}\right)}-\frac{1}{\cos \left(\theta_{1}\right)}$ remove the angular dependancy. This point has been already experimentaly demonstrated [13]. 
The Sample $\operatorname{Al}(20 \mu \mathrm{m}) / \operatorname{Ti}(10 \mu \mathrm{m})$ is irradiated in order to compare the value of $\Delta \mu \cdot d$ with those previously obtained with a $\operatorname{Al}(50 \mu \mathrm{m}) / \operatorname{Ti}(10 \mu \mathrm{m})$ sample [13]. The mean value of $\Delta \mu \cdot d$ (calculated with 3 different couples of angles $\theta_{1}, \theta_{2}$ ) for $20 \mu \mathrm{m}$ aluminium is equal to $-0.35 \pm 0.03$, whereas the mean value was equal to $-0.90 \pm 0.06$ for the thicker sample in [13]. The ratio of this two values, $2.57 \pm 0.09$, is equal to the ratio of the aluminium thicknessses which is equal to $2.5(50 / 20)$. Therefore, results show a linear behavior of $\Delta \mu \cdot d$ with the attenuation layer thickness.

If the emitting element is repeated in several separated layers, equation 3 is no longer valid, and the calculated $\Delta \mu \cdot d$ will vary with the choice of $\theta_{1}$ and $\theta_{2}$. Therefore it's a way to determine if an element is repeated or not. For this purpose a minimum of 3 irradiations with 3 different detection angles are required to check the variation of $\Delta \mu \cdot d$.

\section{Analysis of a multi-layer sample with two layers of the same element}

A sample have been irradiated with a $68 \mathrm{MeV}$ proton beam with three different detection angles. Fig 3 shows one of the obtained spectra. Three el-

ements (titanium, silver and gold) are detected thanks to their characteristic X-rays. 


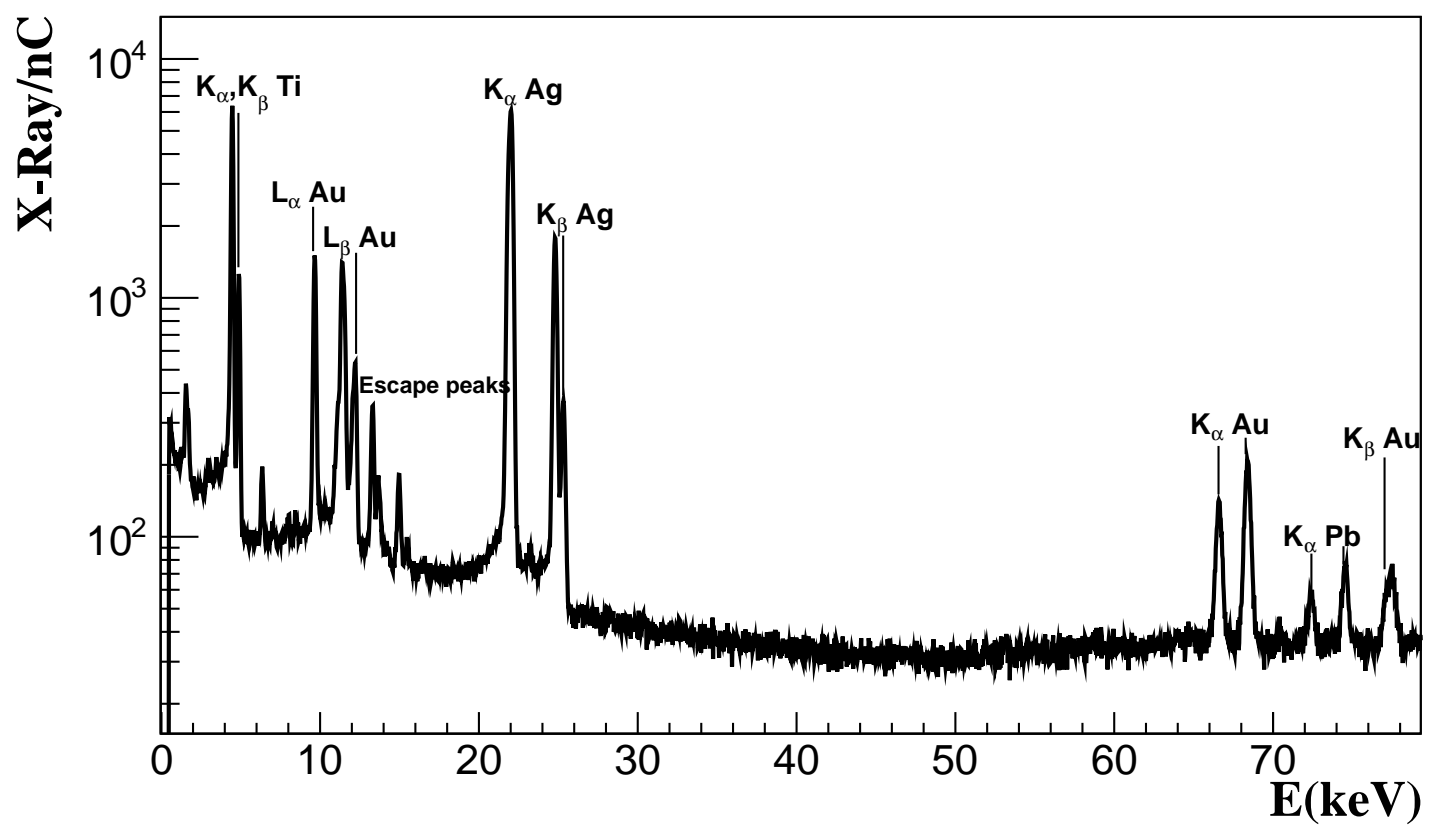

Figure 3: Spectra of the investigated target obtained using $68 \mathrm{MeV}$ proton beam with the detector normal to the target surface $\left(\theta=0^{\circ}\right)$.

For the titanium, the ratio of the $\frac{K_{\alpha}}{K_{\beta}}$ intensity is constant when $\theta$ varies (see table 2). We can then, deduce that there is no layer in front of the titanium, and its thickness is determined (see table 4) using equation 5 without the attenuation correction.

Table 2: Values of $\mathrm{R}$ for the titanium as a function of $\theta$.

\begin{tabular}{|c|c|}
\hline$\theta\left(^{\circ}\right)$ & $\mathrm{R}$ \\
\hline 0 & $5.43 \pm 0.22$ \\
\hline 22 & $5.43 \pm 0.22$ \\
\hline 45 & $5.42 \pm 0.22$ \\
\hline
\end{tabular}

In the gold case, $\mathrm{R}$ (calculated using $L_{\alpha}$ and $K_{\alpha}$ lines) varies with the detection angle so equation 5 is used to determine its thickness (see table 4 ).

For the silver, in contrary to the others layers, $\Delta \mu \cdot d$ is not constant with $\theta$ (see table 3).

Table 3: Values of $\Delta \mu \cdot d$ for the silver as a function of $\theta$. 


\begin{tabular}{|c|c|c|}
\hline$\theta_{1}\left(^{\circ}\right)$ & $\theta_{2}\left(^{\circ}\right)$ & $\Delta \mu \cdot d$ \\
\hline 22 & 0 & $-0.623 \pm 0.009$ \\
\hline 45 & 22 & $-0.104 \pm 0.001$ \\
\hline 45 & 0 & $-0.207 \pm 0.003$ \\
\hline
\end{tabular}

This variation signs more than one silver layer inside the sample. All those information (three elements detected in the spectrum, the titanium is the first layer and the silver is the only element to be repeated) give us the sequences of the layers (see figure 4).

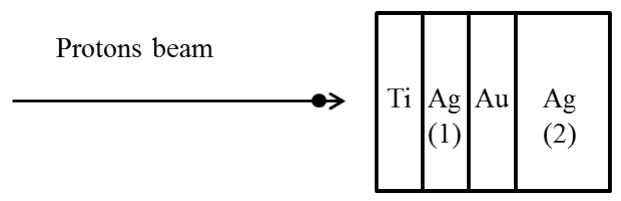

Figure 4: Scheme of the layers sequences inside the irradiated target.

Therefore the following sequence is the only one possible : titanium is the first layer, the second and the last are made of silver and gold is the third layer.

Therefore the attenuation, $A^{A u}$, of the X-ray from the gold layer is the product of the attenuation by the titanium and the first layer of silver and is given by :

$$
A^{A u}=e^{-\mu_{T i} \cdot d_{T i}} \cdot e^{-\mu_{A g_{1}} \cdot d_{A g_{1}}}
$$

Equation 4 is used to calculate $A^{A u}$ from the value of $\Delta \mu \cdot d$. We can calculate the thickness ( see table 4) of the first layer of silver using :

$$
d_{A g_{1}}=\frac{-1}{\mu_{A g}} \cdot \ln \left(\frac{A^{A u}}{e^{-\mu_{T i} \cdot d_{T i}}}\right)
$$

In the spectra, the intensity of the silver X-ray is the sum of the contribution from the two layers. Knowing the thickness of the first layer, we can calculate its X-ray intensity and therefore determine the intensity of the second layer $I_{A g_{2}}$. This intensity, $I_{A g_{2}}$, is then corrected by the attenuation of the other layers (titanium, silver, gold) to determine the second layer thickness by using the same method as for titanium (see table 4).

Table 4: Results of the multi-layers analysis with the repetition of an element. The elements are listed in their real position inside the sample. 


\begin{tabular}{|c|c|c|}
\hline Element & Calculated thickness $(\mu \mathrm{m})$ & Real thickness $(\mu \mathrm{m})$ \\
\hline Titanium & $10.13 \pm 1.23$ & $10.3 \pm 0.02$ \\
Silver $(1)$ & $9.23 \pm 0.91$ & $10.4 \pm 0.02$ \\
Gold & $9.8 \pm 1.08$ & $10.79 \pm 0.02$ \\
Silver $(2)$ & $23.3 \pm 2.4$ & $24.59 \pm 0.01$ \\
\hline
\end{tabular}

The calculated thicknesses presented in table 4 are in good agreement with the real thicknesses of each layers of our samples, even for the repeated layer.

\section{Some limitations to be worked out :}

We were able to determine the thickness of the second silver layer with a small relative error, because its contribution to the total X-ray intensity is significant. In the case of a non repeated layer, using $\Delta \mu \cdot d$ to determine the thickness of each layer [13], the error doesn't depend of the number of layers.

To calculate the thickness of the repeated layers, the thickness of each layer in front of the considered ones is required. The uncertainty calculated using the propagation uncertainty formula will increase with the number of layers in front of the repeated one. The performance of this method in those cases depends on the studied object (number of layers, sequences, thicknesses of the repeated layers).

In more complex cases, with multiple repetition, this method is no longer applicable. But the use at the same time of complementary IBA techniques, such as PIGE or detection of charged particles, could provides additionnal useful information to deal with those more complex cases.

\section{Conclusion}

We demonstrate the possibility to perform quantitative analysis of multilayer targets using high energy PIXE, without any assumption on their composition. A proper study of the parameters involved in the method previously published [13] is presented and more complex cases are investigated. The framework of the method depending on the atomic number of the analyzed element has been defined by looking at the self attenuation factor. We demonstrate the linearity of $\Delta \mu \cdot d$ involved in the thickness calculation.

An extension of this analysis method also based on the relative variation of $\frac{K_{\alpha}}{K_{\beta}}\left(\right.$ or $\left.\frac{K_{\alpha}}{L_{\alpha}}, \frac{L_{\alpha}}{L_{\beta}}\right)$ when the sample is rotated, has been applied to a multilayer sample with a repeated layer. The sequences and the thicknesses of the 
layers without assumption on the sample composition have been determined even for the repeated layer. For samples without any repeated layer, the error on the thickness is low. In the case of a repeated layer, the X-ray intensity has to be significant to apply this method.

The thicknesses determined in this work are in the same order of magnitude as in cultural heritage samples analysed by PIXE. The performance of this method will be tested on real cases and especially on art objects already analysed by HEPIXE in the near future.

\section{Acknowledgments}

The ARRONAX cyclotron is a project promoted by the Regional Council of Pays de la Loire financed by local authorities, the French government and the European Union. This work has been, in part, supported by a grant from the French National Agency for Research called Investissements dAvenir, Equipex Arronax-Plus no ANR-11-EQPX-0004.

\section{Reference}

[1] R. Borges, L. Alves, R. Silva, M. Araújo, A. Candeias, V. Corregidor, P. Valério, P. Barrulas, Investigation of surface silver enrichment in ancient high silver alloys by PIXE, EDXRF, LA-ICP-MS and SEM-EDS, Microchemical Journal 131 (2017) 103-111.

[2] A. Zucchiatti, A. C. Font, P. C. G. Neira, A. Perea, P. F. Esquivel, S. R. Llorens, J. L. R. Sil, A. Verde, Prehispanic goldwork technology study by PIXE analysis, Nuclear Instruments and Methods in Physics Research Section B: Beam Interactions with Materials and Atoms 332 (2014) 160-164.

[3] M. Rizzutto, M. Moro, T. Silva, G. Trindade, N. Added, M. Tabacniks, E. Kajiya, P. Campos, A. Magalhães, M. Barbosa, External-PIXE analysis for the study of pigments from a painting from the Museum of Contemporary Art, Nuclear Instruments and Methods in Physics Research Section B: Beam Interactions with Materials and Atoms 332 (2014) 411414 .

[4] P. Midy, I. Brissaud, Application of a new algorithm to depth profiling by PIXE, Nuclear Instruments and Methods in Physics Research Section B: Beam Interactions with Materials and Atoms 103 (4) (1995) 489-493. 
[5] I. Brissaud, G. Lagarde, P. Midy, Study of multilayers by PIXE technique. Application to paintings, Nuclear Instruments and Methods in Physics Research Section B: Beam Interactions with Materials and Atoms 117 (1-2) (1996) 179-185.

[6] G. Weber, D. Strivay, L. Martinot, H.-P. Garnir, Use of PIXE-PIGE under variable incident angle for ancient glass corrosion measurements, Nuclear Instruments and Methods in Physics Research Section B: Beam Interactions with Materials and Atoms 189 (1) (2002) 350-357.

[7] Ž. Šmit, J. Istenič, T. Knific, Plating of archaeological metallic objectsstudies by differential PIXE, Nuclear Instruments and Methods in Physics Research Section B: Beam Interactions with Materials and Atoms 266 (10) (2008) 2329-2333.

[8] P. Mandò, M. Fedi, N. Grassi, A. Migliori, Differential PIXE for investigating the layer structure of paintings, Nuclear Instruments and Methods in Physics Research Section B: Beam Interactions with Materials and Atoms 239 (1) (2005) 71-76.

[9] A. Denker, K. Maier, Investigation of objects d'art by PIXE with 68 $\mathrm{MeV}$ protons, Nuclear Instruments and Methods in Physics Research Section B: Beam Interactions with Materials and Atoms 161 (2000) 704708.

[10] A. Denker, M. Blaich, PIXE analysis of Middle Age objects using 68 $\mathrm{MeV}$ protons, Nuclear Instruments and Methods in Physics Research Section B: Beam Interactions with Materials and Atoms 189 (1) (2002) 315-319.

[11] T. Dupuis, G. Chene, F. Mathis, A. Marchal, M. Philippe, H.-P. Garnir, D. Strivay, Preliminary experiments: High-energy alpha PIXE in archaeometry, Nuclear Instruments and Methods in Physics Research Section B: Beam Interactions with Materials and Atoms 268 (11) (2010) 1911-1915.

[12] A. Denker, J. Opitz-Coutureau, Paintings-high-energy protons detect pigments and paint-layers, Nuclear Instruments and Methods in Physics Research Section B: Beam Interactions with Materials and Atoms 213 (2004) 677-682. 
[13] A. Subercaze, A. Guertin, F. Haddad, C. Koumeir, V. Métivier, N. Servagent, Thick multi-layers analysis using high energy PIXE, Nuclear Instruments and Methods in Physics Research Section B: Beam Interactions with Materials and Atoms.

[14] F. Haddad, L. Ferrer, A. Guertin, T. Carlier, N. Michel, J. Barbet, J.F. Chatal, ARRONAX, a high-energy and high-intensity cyclotron for nuclear medicine, European Journal of Nuclear Medicine and Molecular Imaging 35 (7) (2008) 1377-1387.

[15] D. Ragheb, C. Koumeir, V. Métivier, J. Gaudillot, A. Guertin, F. Haddad, N. Michel, N. Servagent, Development of a PIXE method at high energy with the ARRONAX cyclotron, Journal of Radioanalytical and Nuclear Chemistry 302 (2) (2014) 895-901.

[16] P. Linstrom, W. Mallard, National Institute of Standards and Technology: Gaithersburg, MD, March 20899.

[17] W. Heitler, The quantum theory of radiation, Courier Corporation, 1954.

[18] I. Orlić, I. Bogdanović, S. Zhou, J. Sanchez, Parametrization of the total photon mass attenuation coefficients for photon energies between $100 \mathrm{eV}$ and $1000 \mathrm{MeV}$, Nuclear Instruments and Methods in Physics Research Section B: Beam Interactions with Materials and Atoms 150 (1) (1999) $40-45$. 